\title{
Phytofabrication of Bioactive Molecules Encapsulated Metallic Silver Nanoparticles from Cucumis sativus L. and Its Enhanced Wound Healing Potential in Rat Model
}

\author{
Perumal Venkatachalam, ${ }^{1}$ Palanivel Sangeetha, ${ }^{1}$ \\ Natesan Geetha, ${ }^{2}$ and Shivendra Vikram Sahi $^{3}$ \\ ${ }^{1}$ Plant Genetic Engineering and Molecular Biology Lab, Department of Biotechnology, Periyar University, \\ Periyar Palkalai Nagar, Salem 636 011, India \\ ${ }^{2}$ Department of Biotechnology, Mother Teresa Women's University, Kodaikanal 624101, India \\ ${ }^{3}$ Department of Biology, Western Kentucky University, Bowling Green, KY 42101-1080, USA \\ Correspondence should be addressed to Perumal Venkatachalam; pvenkatlab@yahoo.in
}

Received 2 March 2015; Revised 18 May 2015; Accepted 20 May 2015

Academic Editor: Yuan Chen

Copyright (C) 2015 Perumal Venkatachalam et al. This is an open access article distributed under the Creative Commons Attribution License, which permits unrestricted use, distribution, and reproduction in any medium, provided the original work is properly cited.

\begin{abstract}
The present study describes a rapid method for synthesis of metallic silver nanoparticles using callus (CAgNPs) and leaf extracts (LEAgNPs) of Cucumis sativus and evaluation of its wound healing activity in rat model. The prepared silver nanoparticles showed a peak at $350 \mathrm{~nm}$ corresponding to the surface plasmon resonance band. The FTIR spectroscopy measurements showed the presence of the possible biomolecules. X-ray diffraction analysis confirmed the crystalline structure of the synthesized silver nanoparticles. TEM images showed the size of the synthesized CAgNPs with diameter ranged from $21 \mathrm{~nm}$ to $23 \mathrm{~nm}$ with polygonal shape whereas, in the case of LEAgNPs, spherical shape was noticed with an average size between $11 \mathrm{~nm}$ and $19 \mathrm{~nm}$. The EDX results indicated the chemical composition at specific locations on synthesized nanoparticles. Furthermore the topical application of ointment prepared using synthesized AgNPs was found to show enhanced wound healing activity in Wistar albino rat model. By the 21st day, the ointment base containing $5 \%(\mathrm{w} / \mathrm{w})$ of silver nanoparticles showed $100 \%$ potential wound healing activity than the standard drug as well as control bases. Results strongly showed that the ointment base containing LEAgNPs was found to be very effective in wound repair mechanism in the experimental rats.
\end{abstract}

\section{Introduction}

Nanoparticle synthesis and characterization is an emerging field of nanobiotechnology, due to their huge applications in the fields of physics, chemistry, biology, and medicine. The synthesis of nanomaterials with the use of green chemicals has vital importance in medicinal and technological aspects. Metallic silver nanoparticles have a wide range of applications in the field of environmental pollution control, drug delivery system, material chemistry, and so on $[1,2]$. Silver nanoparticles provide a powerful platform in biomedical applications of biomolecular recognition, biosensing, drug delivery, and molecular imaging [3,4]. Silver is well known as it is one of the most universal antimicrobial substances and it is highly toxic to microorganism which shows the biocidal effect against microbial species. The chemical synthesis of silver nanoparticles can cause several occupational hazards like carcinogenicity, genotoxicity, cytotoxicity, and general toxicity [5]. Plant extracts have been applied for synthesis of silver nanoparticle which highlights the possibility of rapid synthesis and may also reduce the steps in downstream process, thereby making the cost efficient protocol $[6,7]$. For thousands of years silver has been used as a healing and antibacterial agent throughout the world [8]. As traditionally used physical and chemical methods for synthesis of silver nanoparticle are quite expensive and involve hazardous chemicals, there is a need to develop rapid, nontoxic, and ecofriendly procedure for synthesis of nanoparticles using plant based bioactive compounds via green technology. 
Cucumber (Cucumis sativus L.) belongs to the Cucurbitaceae family; the medicinal properties of cucumber had been well described since ancient times. Different parts of the plants, namely, leaf, fruit, and seeds, have been explored for their therapeutic benefits [9]. It is believed that they promote refreshing, cooling, healing, soothing, emollient, and itching effect to irritated skin [10]. Antihyaluronidase and antielastase activities have been proven for their cosmetic properties [11]. Few bioactive compounds have been derived from this plant belonging to different chemical groups. Bitter principles of cucurbitacins are the characteristic properties of this species [9]. There were very few reports on the wide use of cucumber plant used as folk medicine [12]. In most of the developing world, plants or herbal products have forever played important roles in the treatment of wounds, intestinal problems, coughs, and sneezes.

Wound healing is a complex multifactorial process that results in the contraction and closure of the wound area and restoration of a functional barrier. Repair of injured tissues occurs as a sequence of events, including inflammation, proliferation, and migration of different cell types. Many plant species have been shown to possess therapeutic potential as promoters of wound healing activity. Medicinal plant extracts have been widely used in facilitating wound healing process with a high degree of success rate [13-16]. Earlier reports have described the effect of crude plant extracts on wound healing activity [17]. Most recently, Bhuvaneswari et al. [18] described a method for synthesis of bioactive compound loaded stable silver nanoparticles from aqueous leaf extracts of Naringi crenulata and studied its wound healing activity in experimental rat model. Although different medicinal plant extracts as well as nanoparticles have been tried for the wound healing treatment in the recent past, there were no reports on wound healing activity using either crude extracts or the silver nanoparticles synthesized using Cucumis sativus plant extracts so far. Also effective and potential wound healing agents from medicinal plants are not available at present. In view of the above, there is an urgent need for the development of efficient wound healing agents derived from traditional medicinal plants. The present study was focused on synthesis and characterization of bioactive molecules loaded metallic silver nanoparticles using cucumber callus and leaf extracts and evaluation of its wound healing activity in Wistar albino experimental rat models.

\section{Materials and Methods}

2.1. Plant Materials. Leaves of cucumber were collected from the Greenhouse, Department of Biotechnology, Periyar University, Salem-11. Callus samples were collected from in vitro culture. Fresh leaves of cucumberwere surface washed with running tap water and then rinsed with sterile distilled water to remove the adhering impurities. Silver nitrate was purchased from Merck, Mumbai, India.

2.2. Biosynthesis of Silver Nanoparticles. The preparation of silver nanoparticles was carried out by using two different methods as follows: (I) boiled method: the fresh leaves (5 gm) and callus tissues (5 gm) individually were collected and cut into fine pieces and we added $10 \mathrm{~mL}$ of sterile distilled water for both samples. The samples were allowed to boil at $60^{\circ} \mathrm{C}$ for $10 \mathrm{~min}$. After boiling, extracts were filtered and used for silver nanoparticle synthesis. (II) Centrifuged method: fresh leaves (5 gm) and callus tissues (5 gm) individually were weighed and ground to fine powder by using mortar and pestle and we added $10 \mathrm{~mL}$ of sterile distilled water to the samples subjected to centrifugation at $10,000 \mathrm{rpm}$ for $10 \mathrm{~min}$. After centrifugation, the supernatant was collected and used for silver nanoparticle synthesis. The plant extracts $(10 \mathrm{~mL})$ from boiled as well as centrifuged samples were used as reducing agents for synthesis of silver nanoparticles. An aqueous solution of $1 \mathrm{mM}$ silver nitrate solution $(90 \mathrm{~mL})$ was added for the reduction of silver ions for all the samples and incubated in the dark conditions overnight and the control sample (silver nitrate without adding plant extract) was also maintained. The reduction of silver ions was monitored by the color change of the synthesized nanoparticles.

2.3. Characterization of Synthesized Metallic Silver Nanoparticles. Preliminary characterization of the synthesized metallic silver nanoparticles was carried out using UV-vis spectrophotometer. The reduction of silver ions was monitored by measuring the absorbance of the reaction mixture in a range of wavelength from 200 to $700 \mathrm{~nm}$ using UV-vis spectrophotometer (Systronics 2350 double beam spectrophotometer) to find an absorbance peak. After incubation, the samples were centrifuged at $10,000 \mathrm{rpm}$ for $10 \mathrm{~min}$ and the pellet was dissolved in sterile distilled water and we washed the nanoparticles for 3 times to remove the impurities. Then the centrifuged samples were air dried and used for further characterization.

Both silver nanoparticles (CAgNPs and LEAgNPs) were then subjected to FT-IR spectroscopy measurements using "Perkin-Elmer makes model spectrum RXI" in the transmit mode in the range of $4000-400 \mathrm{~cm}^{-1}$ in $\mathrm{KBr}$ pellets to find the possible functional groups. The X-ray diffraction measurement for biosynthesized AgNPs was carried out on Shimadzu Model XRD 6000 using $\mathrm{Cu} \mathrm{k}_{\alpha}$ radiation which was facilitated at $40 \mathrm{KV}$ and with a wavelength of $1.54187 \mathrm{~nm}$. Subsequently the X-ray patterns were obtained in the $2 \theta$ range of $20^{\circ}-$ $80^{\circ}$. The size and morphology of the synthesized AgNPs were studied using transmission electron microscopy (TEM, Carl Zeiss Ultra 55 model) and the size of the nanoparticles was determined using the Sigma Scan Pro Software. Furthermore, the presence of elemental silver region was confirmed by Xray energy dispersive spectrometer (EDAX) attached with TEM.

2.4. Preparation of AgNPs Based Ointment. A simple ointment was prepared by trituration in a ceramic mortar and pestle using white soft paraffin base. Three batches of the simple ointment (Batches A, B, and C) were prepared and used for wound healing experiments. Batch A ointment was prepared without any nanoparticles and standard drug. Batch B ointment contains 5\% w/w Betadine (standard drug) and Batch C ointment consists of synthesized LEAgNPs. These ointments were prepared as per Indian pharmacopeia by fusion method. 
TABLE 1: Effect of externally applied green synthesized LEAgNPs based ointment on excision wound model in experimental rats.

\begin{tabular}{|c|c|c|c|c|c|c|c|c|}
\hline \multirow{2}{*}{ Treatment groups } & \multicolumn{8}{|c|}{ Wound contraction $\left(\mathrm{mm}^{2}\right)$ on day $\pm \mathrm{SE}$ and percentage of wound contraction } \\
\hline & Day 0 & Day 3 & Day 6 & Day 9 & Day 12 & Day 15 & Day 18 & Day 21 \\
\hline Control & $507.64 \pm 0.70$ & $474.60 \pm 4.72$ & $398.76 \pm 3.29$ & $362.73 \pm 5.87$ & $282.09 \pm 6.63$ & $204.8 \pm 10.12$ & $95.39 \pm 4.68$ & $33.11 \pm 2.90$ \\
\hline Std. drug & $506.48 \pm 2.38$ & $462.52 \pm 2.36$ & $350.14 \pm 11.5$ & $298.57 \pm 3.56$ & $177.7 \pm 5.71$ & $80.33 \pm 9.95$ & $18.48 \pm 2.09^{*}$ & $5.17 \pm 1.35^{*}$ \\
\hline AgNPs & $508.34 \pm 1.20$ & $415.48 \pm 5.52$ & $329.84 \pm 3.49$ & $262.10 \pm 7.26$ & $138.1 \pm 3.12$ & $16.16 \pm 2.06^{*}$ & $2.33 \pm 0.59^{*}$ & $0 \pm 0.00$ \\
\hline
\end{tabular}

${ }^{*}$ The values are mean \pm SEM $(n=4)$ with statistically significant difference in comparison with control group: $p<0.05$.

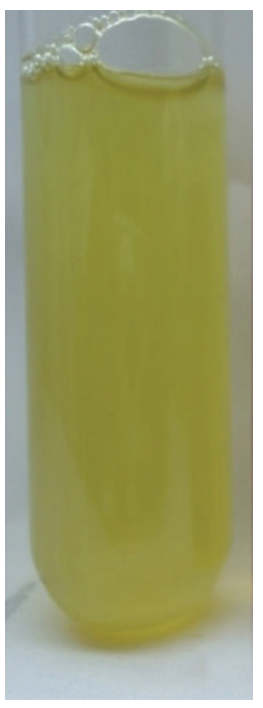

(a)

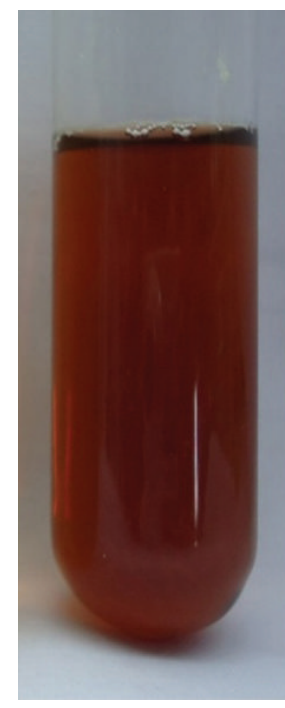

(b)

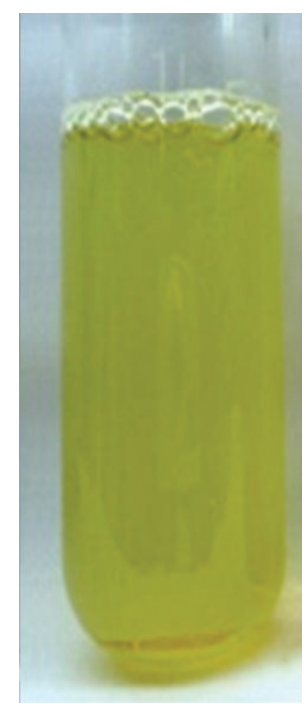

(c)

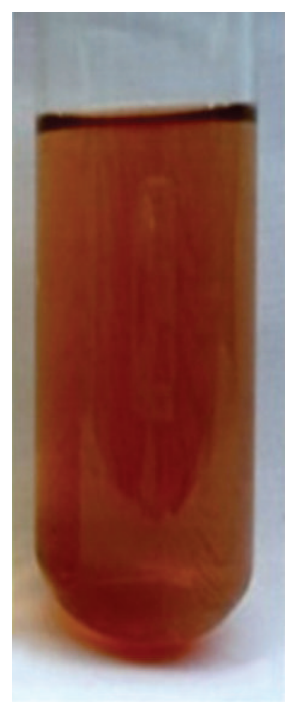

(d)

Figure 1: Visual observations of color change in the reaction mixtures: (a) callus extract, (b) CAgNPs, (c) leaf extract, and (d) LEAgNPs.

\subsection{Wound Healing Activity}

2.5.1. Experimental Animals. Healthy adult male Wistar rats (150-200 g) were randomly selected for the present in vivo studies. Rats were housed individually in polypropylene cages in well ventilated environmental conditions (temperature of $25 \pm 2^{\circ} \mathrm{C}$, relative humidity of $55 \pm 10 \%$, and $12: 12$ light : dark cycle). The rats were fed with standard pellet diet (Amrut rat and mice feed, Sangli, India) and water ad libitum. All the experiments were conducted in accordance with the approval of the Institutional Animal Ethics Committee, Mother Teresa Women's University, Kodaikanal (IAEC Approval Number 001/BT/MTWU/IAEC/0209/2013/02 and CPCSEA Registration Number 933/PO/c06/CPCSEA).

2.5.2. Excision Wound Healing. The rats were anesthetized with diethyl ether before and during the infliction of experimental wounds. The hairs were removed by shaving the back of all the rats and ethanol (70\%) was applied on the region before making the wound. Excision wound of $500 \mathrm{~mm}^{2}$ was created on the depilated dorsal thoracic region of the rats. After wound creation, experimental animals were divided into the following three groups, each group consisting of 6 rats.

Group 1: negative control was treated with blank ointment formulation (Batch A). Group II: wounds were treated topically with standard drug Betadine as positive control
(Batch B). Group III: wounds were treated with an ointment base prepared with synthesized silver nanoparticles using centrifuged leaf extracts of cucumber (Batch C).

2.5.3. Wound Contraction and Epithelization Period. The ointment was topically applied daily once until the wounds were completely healed. Wound area was measured at day zero $\left(500 \mathrm{~mm}^{2}\right)$ and once every 3 days after wounding. Excision wound margins were measured on 3rd, 6th, 9th, 12th, 15th, 18th, and 21st day after treatment using transparent paper. The tracing of the wound was then shifted to graph paper, and the measured wound surface area at different days after wound creation was employed to calculate the percentage of wound contraction which was expressed as unit $\left(\mathrm{mm}^{2}\right)$ percentage reduction of original wound size (Table 1).

2.6. Statistical Analysis. Data were expressed as a mean \pm standard error. Statistical analysis was carried out using one-way ANOVA and differences in mean between paired observations were considered statistically significant when $p$ values were $<0.05$.

\section{Results and Discussion}

3.1. Biosynthesis of Silver Nanoparticles. In the current study, the cucumber plant extracts were prepared adopting two 


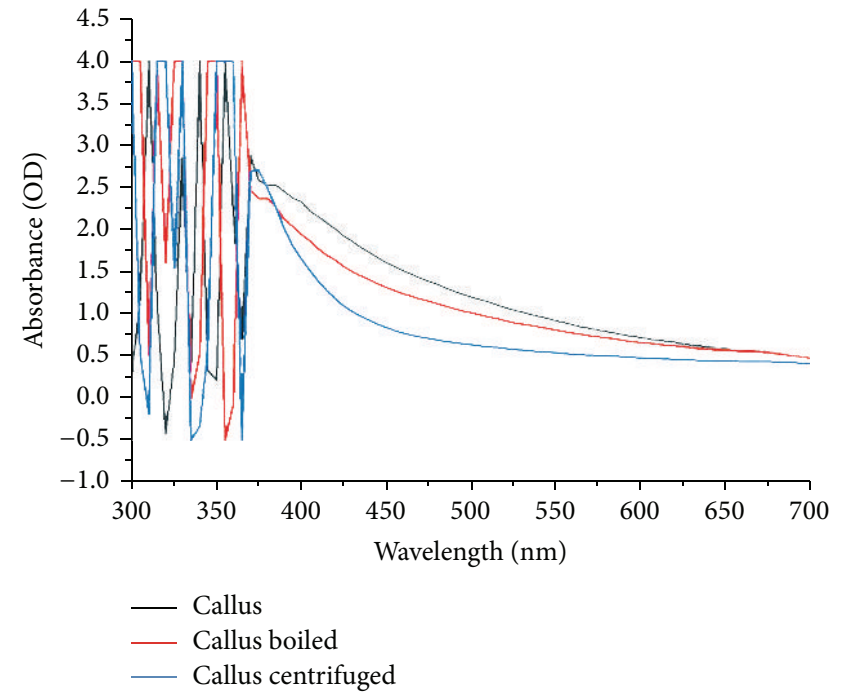

(a)

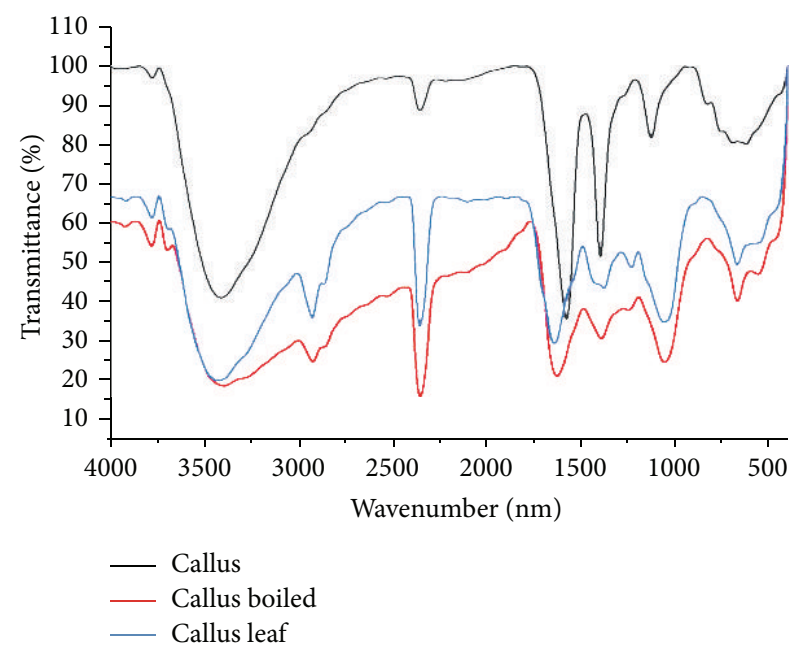

(c)

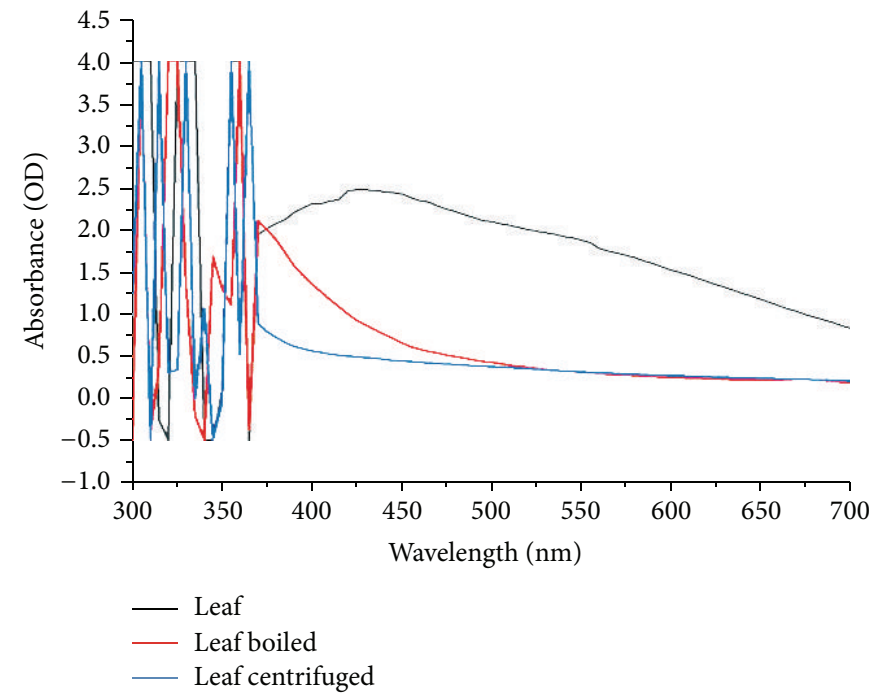

(b)

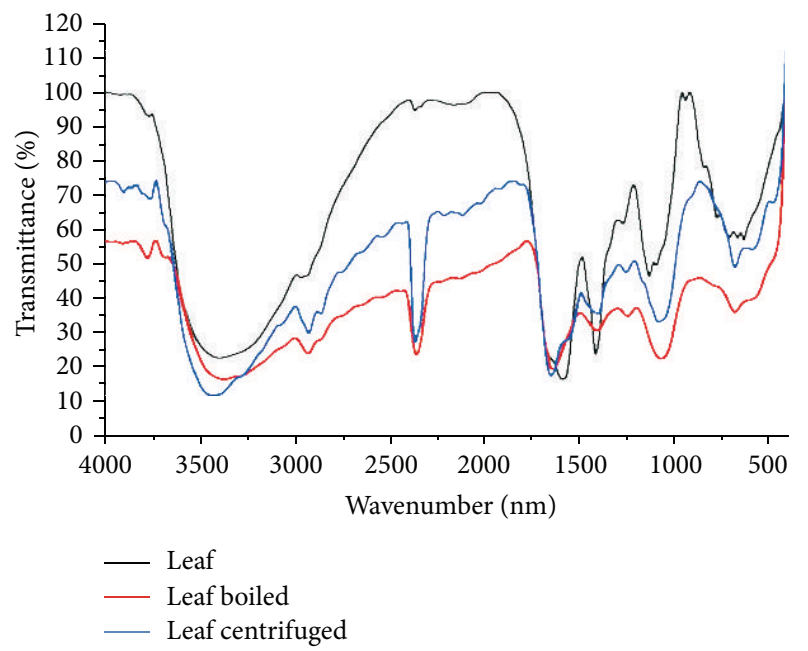

(d)

FIGURE 2: UV-vis spectroscopy analysis in the range of 300-700 nm for AgNPs synthesis: (a) CAgNPs and (b) LEAgNPs and FT-IR spectral analysis of biosynthesized AgNPs: (c) CAgNPs and (d) LAgNPs.

methods for extracellular synthesis of metallic silver nanoparticles (CAgNPs and LEAgNPs). Both the callus and leaf extracts were mixed with an aqueous solution of silver nitrate resulting in color change from green to reddish brown depending on the intensity and the size of nanoparticles. The color change was due to the excitation of surface plasmon resonance with the AgNPs $[5,19]$. The color change was recorded by visual observation in both callus and leaf extracts when incubated with $\mathrm{AgNO}_{3}$ solution (Figures 1(a)-1(d)). The color intensity was found to be higher with centrifuged extract than the boiled extract. Therefore, further characterization was carried out with the AgNPs synthesized using centrifuged extracts (both callus and leaf samples) only.

\subsection{Characterization of Synthesized Silver Nanoparticles}

3.2.1. UV-Vis Spectroscopy. UV-visible spectroscopy is one of the valuable techniques for structural characterization and stabilization of silver nanoparticles. It is known that silver nanoparticles are expected to exhibit a UV-visible absorption peak maximum in the range of $300-500 \mathrm{~nm}$. The reduction of pure $\mathrm{Ag}^{+}$ions to $\mathrm{Ag}^{0}$ was monitored by measuring UVvisible spectrum of the reaction media at regular intervals. In the present study, the silver surface plasmon resonance was observed at $350 \mathrm{~nm}$ showing shift of the maximum wavelength. An increased intensity could be due to increasing of the number of nanoparticles produced as a result of reduction of silver ions present in the aqueous medium. Earlier report confirmed that maximum absorbance occurred due to presence of silver particles [20]. The absorbance spectrum area increased with the increase in reaction time. The possible reason for this result could be due to the bioreduction of silver ion by the presence of biomolecules in the leaf extracts [7]. The control solutions of cucumber callus or leaf extract or $1 \mathrm{mM} \mathrm{AgNO}$ neither developed the characteristic brown colors nor did they display the 


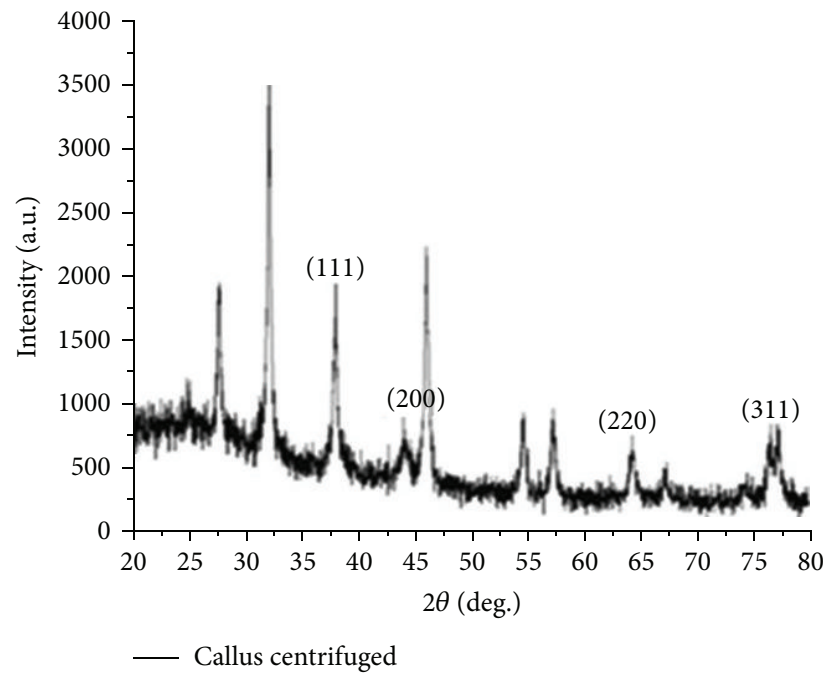

(a)

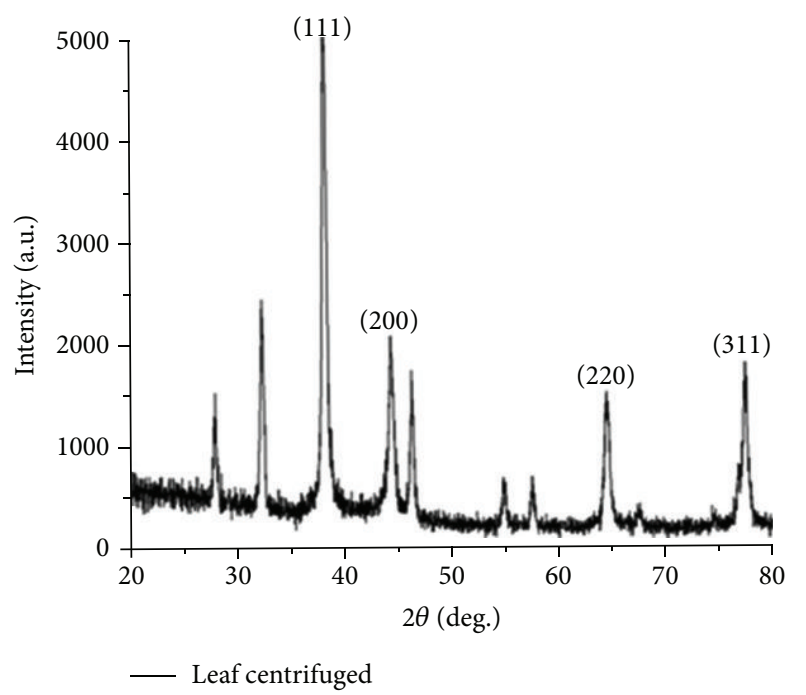

(b)

FIGURE 3: XRD pattern exhibiting the facets of crystalline nature of synthesized AgNPs: (a) CAgNPs and (b) LAgNPs.

characteristic peaks in the present study. Results confirmed that the reduction of $\mathrm{AgNO}_{3}$ did not occur under the reaction conditions. Similar results were also demonstrated recently $[18,21,22]$. It is well recognized that the optical absorption peak of metal nanoparticles was dominated by surface plasmon resonances (SPRs) that shift to longer wavelengths with increasing particle size due to the capping agent [23, 24]. It is reported that the SPR was mainly depending upon the size and shape of AgNPs [25]. In general, the number of SPR spectra decreases as the symmetry of the nanoparticle increases [26]. The UV-vis spectrum was also recorded after $24 \mathrm{~h}$ incubation showed that there was no increase in the absorption spectra which confirms the complete synthesis of silver nanoparticles (Figures 2(a) and 2(b)). The SPR absorbance was extremely sensitive to the nature, size and shape of the particles formed, their interparticle distances, and the surrounding media. Pimprikar et al. [27] described the influence of level of active biomolecules present in the biological samples on nanoparticle formation.

\subsubsection{Fournier Transform Infrared Spectroscopy. FT-IR} spectroscopy analysis was characterized to identify the biomolecules that were bound specifically on the synthesized AgNPs. Synthesized silver nanoparticles using centrifuged callus extracts (CAgNPs) showed peaks at 3426, 2926, 2103, 1896, 1639, 1232, 1059, and $669 \mathrm{~cm}^{-1}$ (Figures 2(c) and 2(d)). The peaks produced at $3426 \mathrm{~cm}^{-1}$ represent the $\mathrm{OH}$ stretching (binding of silver ions with groups) which contains alcohols and phenols. The spectra at $2926 \mathrm{~cm}^{-1}$ denote $\mathrm{CH}$ stretching which reveals the presence of alkenes group. The band at $2103 \mathrm{~cm}^{-1}$ stretches alkynes group. The peak at $1869 \mathrm{~cm}^{-1}$ stretches $\mathrm{CH}$ which contains aromatic group. The peak at $1639 \mathrm{~cm}^{-1}$ represents $\mathrm{C}-\mathrm{C}$ stretches, indicating the presence of alkenes. The spectra at $1232 \mathrm{~cm}^{-1}$ indicate CO stretching alcohols, carboxylic acids, esters, and ethers. The peak at $1059 \mathrm{~cm}^{-1}$ stretches $\mathrm{C}-\mathrm{N}$ containing aliphatic amines. Synthesized silver nanoparticles by centrifuged leaf extracts (LEAgNPs) showed peaks at 3764, 3433, 2923, 2859, 2359, 2206, 2110,1639,1392,1243,1070,667, and $465 \mathrm{~cm}^{-1}$ (Figure 2(b)). The peak produced at $3764 \mathrm{~cm}^{-1}$ indicates N-H stretches amide group. The peak at $3433 \mathrm{~cm}^{-1}$ represents the $\mathrm{OH}$ stretching (binding of silver ions with $\mathrm{OH}$ groups) phenols and alcohols. The band at $2859 \mathrm{~cm}^{-1}$ stretches $\mathrm{C}-\mathrm{H}$ indicates the presence of alkanes group, while at $2359 \mathrm{~cm}^{-1}$ stretch with $\mathrm{O}-\mathrm{H}$ group represents the presence of carboxylic acids, and peaks at 2206, $2110 \mathrm{~cm}^{-1}$ with $\mathrm{C}-\mathrm{C}$ stretch confirms the presence of alkynes group. The peaks at $1243,1070 \mathrm{~cm}^{-1}$ represent $\mathrm{C}-\mathrm{N}$ stretching aliphatic amine groups. The bands appearing at $667,465 \mathrm{~cm}^{-1}$ are due to the presence of alkyl halides (C-Br) stretching. These abovementioned peaks clearly showed that the active biomolecules involved in the reduction and capping of silver ions were different in both extracts. The cucumber leaf extracts are rich resources of phytoconstituents, like cucurbitacin, flavonoids, and phenols that were capped on synthesized AgNPs. Macromolecules such as proteins could easily bind and interact with silver salts through either free amine groups or cysteine residues that are ultimately involved in stabilization of the synthesized silver nanoparticles [22]. As mentioned earlier, amine and phenol groups were the surface active molecules present in the leaf extracts and participated in the reduction of metal ions and stabilization of nanoparticles in the reaction mixture $[22,28]$ and the protein secondary structures were altered after reaction of Capsicum annuum L. extract with silver ions [29]. These results together suggested that the functional groups of these bioactive compounds proved to have potential to act as reducing and stabilizing agents during the synthesis of silver nanoparticles in this study.

3.2.3. X-Ray Diffraction Analysis. The exact nature of the AgNPs synthesized was studied through XRD analysis. The 


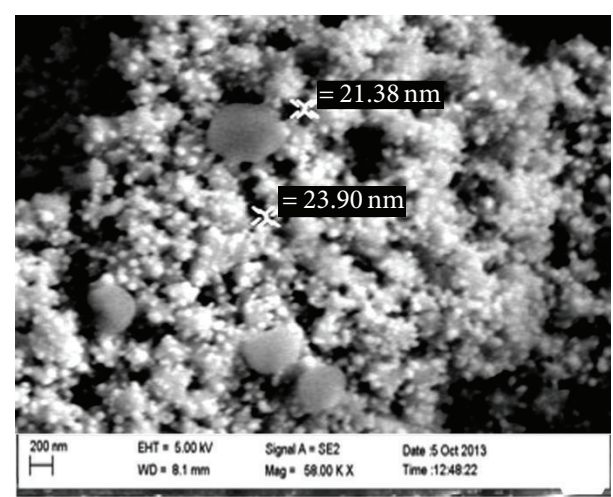

(a)

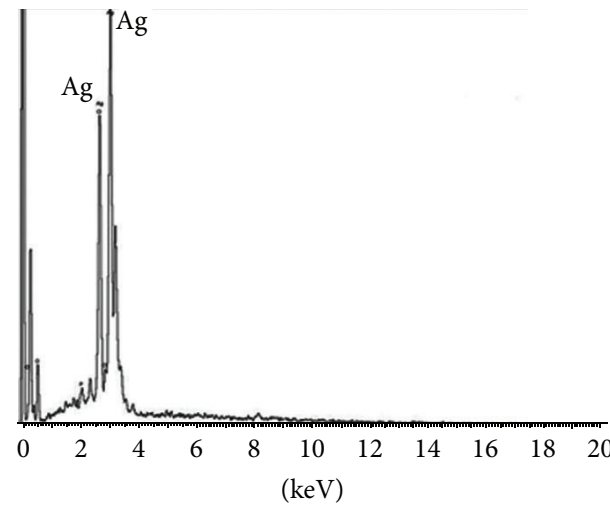

(c)

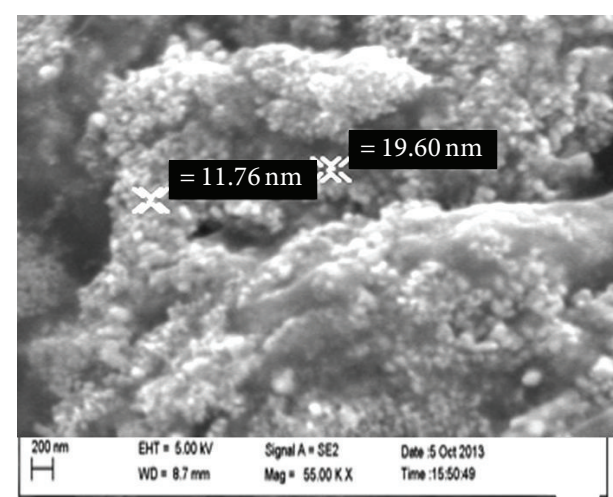

(b)

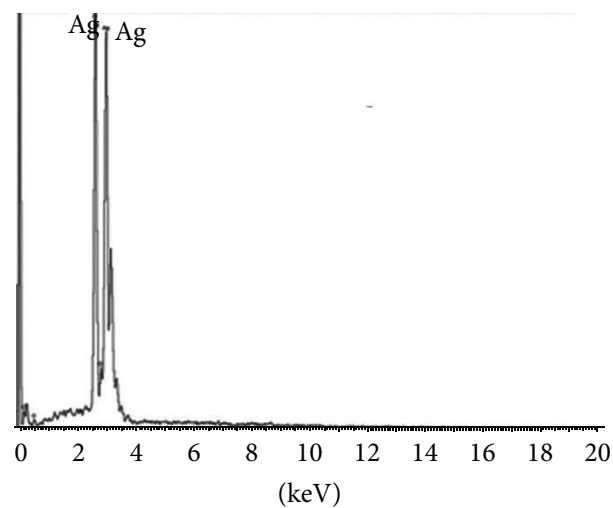

(d)

FIgure 4: TEM images of synthesized AgNPs: (a) CAgNPs and (b) LAgNPs and EDX spectrum exhibited the strong signal of silver from synthesized nanoparticles: (c) CAgNPs and (d) LEAgNPs.

result clearly showed strong and narrow diffraction peaks that indicated the synthesized AgNPs were found to be crystalline in nature (Figures 3(a) and 3(b)). A number of Bragg's reflection peaks were noticed at $2 \theta$ values of $38.116^{\circ}, 44.277^{\circ}$, $64.426^{\circ}$, and $77.472^{\circ}$ corresponding to the (111), (200), (220), and (311) planes for callus extracts of cucumber and reflection peaks observed at $2 \theta$ values of $38.116^{\circ}, 44.277^{\circ}, 64.426^{\circ}$, and $77.472^{\circ}$ corresponding to (111), (200), (220), and (311) planes for leaf extracts. The peaks indicate the face-centered cubic (fcc) structure of AgNPs, which are closely linked with JCPDS (file number 04-0783) for silver. The results of XRD peaks strongly reveal the synthesis of silver nanoparticles with sharp bands of Bragg peaks, and this may due to the stabilization of the synthesized nanoparticles by the reducing agents present in the callus and leaf extract and provides the crystalline nature of the silver nanoparticles [30]. The present results clearly suggested that the synthesized AgNPs were crystalline in nature due to the presence of bioactive molecules in plant extracts.

3.2.4. FESEM and EDAX Analysis. TEM images illustrated the shape and the size of the synthesized AgNPs. According to the TEM micrograph, the surface morphology of the synthesized silver nanoparticle noticed was spherical and polygonal shapes (Figures $4(\mathrm{a})$ and $4(\mathrm{~b})$ ). The average size of the CAgNPs with diameter ranged from $21 \mathrm{~nm}$ to $23 \mathrm{~nm}$ whereas the sizes of the LEAgNPs were found to be in the range of 11-19 nm. Most of the synthesized nanoparticles were found to be aggregated and only a few of them were scattered, as observed under TEM. Xu and Käll [31] demonstrated that the shape of metal nanoparticles considerably altered their optical and electronic properties due to the presence of various bioactive molecules. The presence of metallic silver in the synthesized nanocrystals was further authenticated by EDAX analysis. The EDX results indicated the chemical composition at specific locations on the synthesized CAgNPs and LEAgNPs (Figures 4(c) and 4(d)). The EDAX spectrum of AgNPs showed a strong signal from the silver atoms and weaker signals from oxygen and other atoms. Results clearly suggested that the sharp spectrum of silver atom confirmed the reduction of silver nitrate to silver nanoparticles. Metallic silver nanocrystals showed strong absorption spectra in the range of $2.5-4 \mathrm{keV}$. Similar results were also reported by Gardea-Torresdey et al. [32] and Vijayakumar et al. [33]. Recently Jeeva et al. [22] obtained AgNPs with spherical shape showing strong absorption spectra in the range of 2.5$4 \mathrm{keV}$ by using $C$. coriaria leaf extracts and Bhuvaneswari et al. [18] also noticed spherical shape metallic silver nanocrystals that showed strong absorption peak in the range of 2.5$4 \mathrm{keV}$ using $N$. crenulata leaf extracts. The strong spectrum signals were likely to be due to the X-ray emission from active biomolecules available in the plant extracts $[22,34]$. 

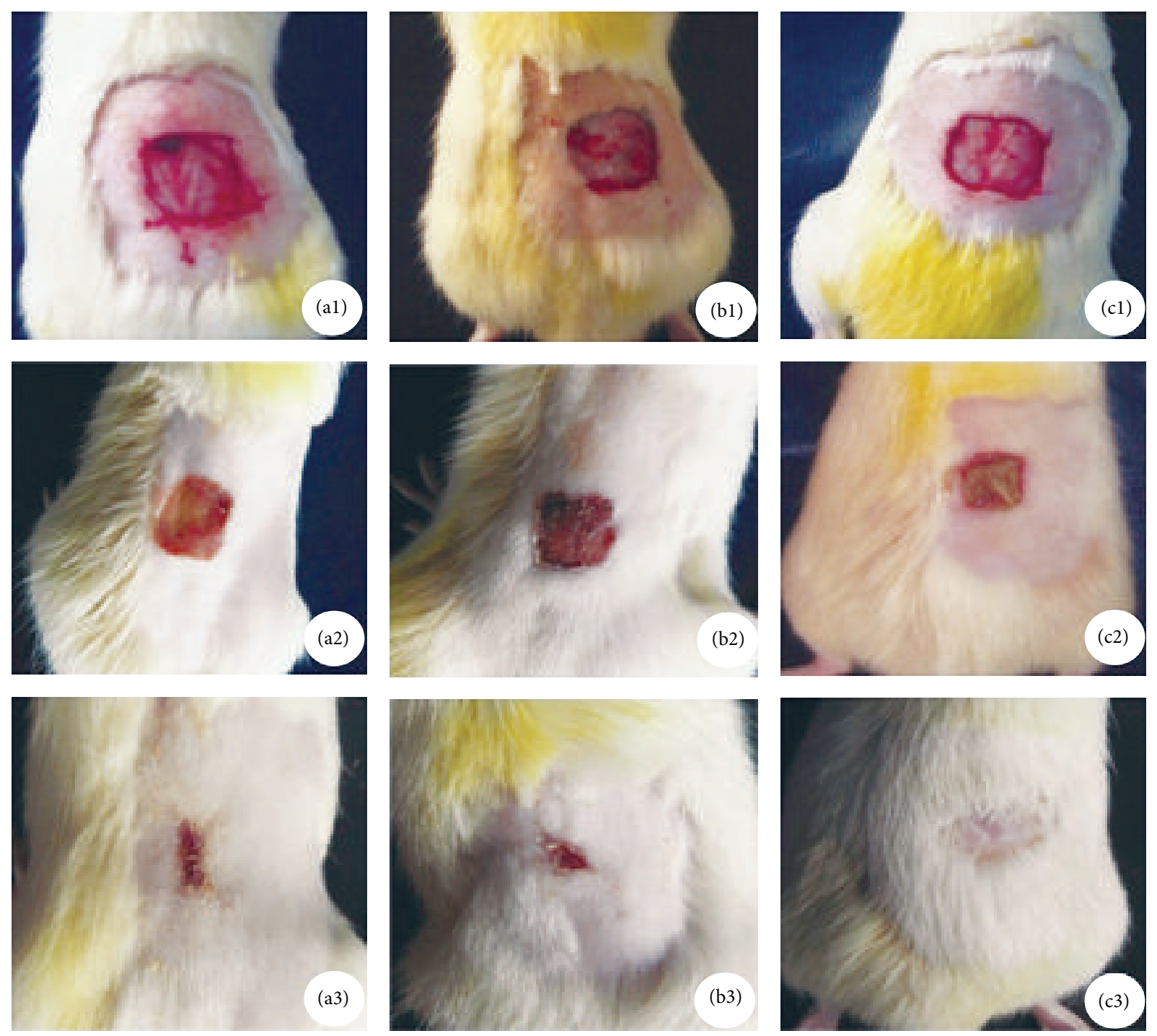

Figure 5: Progress of cutaneous wound healing in negative-control rats untreated (a1-a3), positive control rats treated with standard drug Betadine ointment (b1-b3), and experimental rats treated with biomolecules loaded LEAgNPs based ointment (cl-c3). Wounds of the dorsal skin before and after treatment without (al-a3), and with (b1-b3, cl-c3) ointments.

3.3. Evaluation of Wound Healing Activity. For wound healing activities, the male Wistar albino rats were randomly divided into 3 groups of six rats in each group. Rats from Group I served as negative control treated with an ointment base (Batch A) while Group II rats acted as positive control treated with standard drug (Betadine, Batch B) and the formulated ointment base containing synthesized silver nanoparticles (LEAgNPs) (Batch C) was applied on Group III rats. The excised wound healing area was measured on 3rd, 6th, 9th, 12th, 15th, 18th, and 21st days and the wound healing area was measured accordingly. After excision of wound, there was no death observed for any of the rats in the study groups and no remarkable changes were noticed in animal behaviour. The area of wound contraction with Batch A ointment treatment was reduced to $474 \mathrm{~mm}^{2}$ of the original size $\left(500 \mathrm{~mm}^{2}\right)$ on day $3,282 \mathrm{~mm}^{2}$ on day 12 , and $33 \mathrm{~mm}^{2}$ on day 21 [Figures 5(a1-a3)]. The area of wound contraction in Batch B ointment (standard drug) was reduced to $462 \mathrm{~mm}^{2}$ of the original size $\left(500 \mathrm{~mm}^{2}\right)$ on day $3,177 \mathrm{~mm}^{2}$ on day 12 , and $5 \mathrm{~mm}^{2}$ on day 21 [Figures $5(\mathrm{bl}-\mathrm{b} 3)$ ]. And the area of wound contraction in Batch C ointment (nanoparticle based ointment) was reduced to $415 \mathrm{~mm}^{2}$ of the original size $\left(500 \mathrm{~mm}^{2}\right)$ on day $3,138 \mathrm{~mm}^{2}$ on day 12 , and $0 \mathrm{~mm}^{2}$ on day 21 [Figures 5(c1-c3)]. There was complete epithelization with nanoparticle based ointment treated rats when compared to untreated group and Betadine treated group and the least wound healing activity was noticed in control compared to the standard drug (Figure 5). It is noteworthy to mention that the topical application of the silver nanoparticles incorporated ointment base (Batch $C$ ) showed a significant $(p<0.05)$ rate of wound healing activity in rats compared to that of control and standard. The time for wound closure was found lesser, and the percentage of wound healing area diameter noticed was much more with the ointment containing silver 
nanoparticles treated group rats (21 days for $100 \%$ contraction). The results of this study clearly showed enhanced levels of wound contraction in experimental rats treated with ointment base containing the synthesized LEAgNPs which may be due to the presence of cucurbitacin (bioactive molecule) in the synthesized AgNPs. The reason could be due to the presence of cucurbitacin triterpenoid substances which are well known for their bitterness and toxicity in the synthesized AgNPs [9]. Akhtar et al. [35] developed a topical skin care cream with cucumber plant, which increases the transepidermal water loss which strengths the antiacne effects. Wound healing activity was reported by using various plant extracts including Kalanchoe petitiana [36] and Flabellaria paniculata [37] and by using AgNPs derived from Naringi crenulata [18] in the recent past. The present wound healing activity results showed that cucumber bioactive molecules loaded LEAgNPs ointment base was found to be potential for effective epithelial wound repair mechanism and softens the skin in experimental rats, due to the presence of a rejuvenating agent in cucumber than other plant species used earlier.

\section{Conclusion}

In conclusion, the present study highlighted the synthesis and characterization of silver nanoparticles from Cucumis sativus L. and wound healing activity of the nanobased ointment application on experimental rats. An effective method for synthesis of metallic silver nanoparticles using callus and leaf extracts of cucumber was established and synthesized AgNPs were characterized by UV-vis spectroscopy, FT-IR, XRD, FESEM, and EDAX. FT-IR results confirmed the presence of possible functional groups in the synthesized silver nanoparticles. XRD peaks indicated that the synthesized silver nanoparticles were highly stable and crystalline in nature for silver. TEM results revealed that the obtained silver nanoparticles were spherical and polygonal in shape. EDAX analysis confirms the presence of elemental silver. In the present study, the nanobased ointment had facilitated $100 \%$ potential wound healing activity within 21 days in experimental rats and increased breaking strength of the repaired tissues. Therefore, the nanobased ointment could be used in the pharmaceutical industry in the near future. The present green biosynthesis of AgNPs protocol using plant extracts has several advantages over microorganisms: (1) sustainable supply of materials for large scale synthesis of NPs; (2) plant extract not containing toxic substances, and (3) variety of bioactive molecules available in different plant species. To the best of our knowledge, this is the first report on formulation of nanobased ointment from cucumber plant species and its efficacy against wound healing potential in rat model.

\section{Conflict of Interests}

The authors declare that there is no conflict of interests regarding the publication of this paper.

\section{Acknowledgment}

The authors' sincere thanks are due to University Grants Commission, Government of India, New Delhi, for providing UGC-BSR Research Fellowship (URF) to Palanivel Sangeetha.

\section{References}

[1] D. I. Gittins, D. Bethell, D. J. Schiffrin, and R. J. Nichols, "A nanometre-scale electronic switch consisting of a metal cluster and redox-addressable groups," Nature, vol. 408, no. 6808, pp. 67-69, 2000.

[2] M. Sathishkumar, K. Sneha, and Y.-S. Yun, "Immobilization of silver nanoparticles synthesized using Curcuma longa tuber powder and extract on cotton cloth for bactericidal activity," Bioresource Technology, vol. 101, no. 20, pp. 7958-7965, 2010.

[3] S. L. Smitha, K. M. Nissamudeen, D. Philip, and K. G. Gopchandran, "Studies on surface plasmon resonance and photoluminescence of silver nanoparticles," Spectrochimica Acta Part A: Molecular and Biomolecular Spectroscopy, vol. 71, no. 1, pp. 186190, 2008.

[4] P. Mohanpuria, N. K. Rana, and S. K. Yadav, "Biosynthesis of nanoparticles: technological concepts and future applications," Journal of Nanoparticle Research, vol. 10, no. 3, pp. 507-517, 2008.

[5] P. Mukherjee, M. Roy, B. P. Mandal et al., "Green synthesis of highly stabilized nanocrystalline silver particles by a nonpathogenic and agriculturally important fungus T. asperellum," Nanotechnology, vol. 19, no. 7, Article ID 075103, pp. 75-103, 2008.

[6] S. S. Shankar, A. Ahmad, and M. Sastry, "Geranium leaf assisted biosynthesis of silver nanoparticles," Biotechnology Progress, vol. 19, no. 6, pp. 1627-1631, 2003.

[7] J. Huang, Q. Li, D. Sun et al., "Biosynthesis of silver and gold nanoparticles by novel sundried Cinnamomum camphora leaf," Nanotechnology, vol. 18, no. 10, Article ID 105104, 2007.

[8] A. Panácěk, L. Kvítek, R. Prucek et al., "Silver colloid nanoparticles: synthesis, characterization, and their antibacterial activity," The Journal of Physical Chemistry B, vol. 110, no. 33, pp. 16248$16253,2006$.

[9] P. K. Mukherjee, N. K. Nema, N. Maity, and B. K. Sarkar, "Phytochemical and therapeutic potential of cucumber," Fitoterapia, vol. 84, no. 1, pp. 227-236, 2013.

[10] P. Franco, S. Vittorio, and A. Robert, Plants in Cosmetics, PressCouncil of Europe, 2002.

[11] N. K. Nema, N. Maity, B. Sarkar, and P. K. Mukherjee, “Cucumis sativus fruit-potential antioxidant, anti-hyaluronidase, and anti-elastase agent," Archives of Dermatological Research, vol. 303, no. 4, pp. 247-252, 2011.

[12] T. Aburjai and F. M. Natsheh, "Plants used in cosmetics," Phytotherapy Research, vol. 17, no. 9, pp. 987-1000, 2003.

[13] C. O. Esimone, C. S. Nworu, and C. L. Jackson, "Cutaneous wound healing activity of a herbal ointment containing the leaf extract of Jatropha curcas L. (Euphorbiaceae)," International Journal of Applied Research in Natural Products, vol. 1, no. 4, pp. $1-4,2008$.

[14] F. Mesfin, S. Demissew, and T. Teklehaymanot, "An ethnobotanical study of medicinal plants in Wonago Woreda, SNNPR, Ethiopia," Journal of Ethnobiology and Ethnomedicine, vol. 5, article 28, 2009. 
[15] C. Muthu, M. Ayyanar, N. Raja, and S. Ignacimuthu, "Medicinal plants used by traditional healers in Kancheepuram District of Tamil Nadu, India," Journal of Ethnobiology and Ethnomedicine, vol. 2, article 43, 2006.

[16] G. N. Njoroge and R. W. Bussmann, "Ethnotherapeautic management of skin diseases among the Kikuyus of Central Kenya," Journal of Ethnopharmacology, vol. 111, no. 2, pp. 303-307, 2007.

[17] J. Somboonwong, M. Kankaisre, B. Tantisira, and M. H. Tantisira, "Wound healing activities of different extracts of Centella asiatica in incision and burn wound models: an experimental animal study," BMC Complementary and Alternative Medicine, vol. 12, article 103, 2012.

[18] T. Bhuvaneswari, M. Thiyagarajan, N. Geetha, and P. Venkatachalam, "Bioactive compound loaded stable silver nanoparticle synthesis from microwave irradiated aqueous extracellular leaf extracts of Naringi crenulata and its wound healing activity in experimental rat model," Acta Tropica, vol. 135, no. 1, pp. 5561, 2014.

[19] P. Prakash, P. Gnanaprakasam, R. Emmanuel, S. Arokiyaraj, and M. Saravanan, "Green synthesis of silver nanoparticles from leaf extract of Mimusops elengi, Linn. for enhanced antibacterial activity against multi drug resistant clinical isolates," Colloids and Surfaces B: Biointerfaces, vol. 108, pp. 255-259, 2013.

[20] M. Sathishkumar, K. Sneha, S. W. Won, C. W. Cho, S. Kim, and Y. S. Yun, "Cinnamon zeylanicum bark extract and powder mediated green synthesis of nano-crystalline silver particles and its bactericidal activity," Colloids and Surfaces B: Biointerfaces, vol. 73, no. 2, pp. 332-338, 2009.

[21] U. B. Jagtap and V. A. Bapat, "Green synthesis of silver nanoparticles using Artocarpus heterophyllus Lam. seed extract and its antibacterial activity," Industrial Crops and Products, vol. 46, pp. 132-137, 2013.

[22] K. Jeeva, M. Thiyagarajan, V. Elangovan, N. Geetha, and P. Venkatachalam, "Caesalpinia coriaria leaf extracts mediated biosynthesis of metallic silver nanoparticles and their antibacterial activity against clinically isolated pathogens," Industrial Crops and Products, vol. 52, pp. 714-720, 2014.

[23] R. Brause, H. Möltgen, and K. Kleinermanns, "Characterization of laser-ablated and chemically reduced silver colloids in aqueous solution by UV/VIS spectroscopy and STM/SEM microscopy," Applied Physics B: Lasers and Optics, vol. 75, no. 6-7, pp. 711-716, 2002.

[24] C. R. K. Rao and D. C. Trivedi, "Biphasic synthesis of fatty acids stabilized silver nanoparticles: role of experimental conditions on particle size," Materials Chemistry and Physics, vol. 99, no. 2-3, pp. 354-360, 2006.

[25] J. J. Mock, M. Barbic, D. R. Smith, D. A. Schultz, and S. Schultz, "Shape effects in plasmon resonance of individual colloidal silver nanoparticles," Journal of Chemical Physics, vol. 116, no. 15, pp. 6755-6759, 2002.

[26] I. O. Sosa, C. Noguez, and R. G. Barrera, "Optical properties of metal nanoparticles with arbitrary shapes," Journal of Physical Chemistry B, vol. 107, no. 26, pp. 6269-6275, 2003.

[27] P. S. Pimprikar, S. S. Joshi, A. R. Kumar, S. S. Zinjarde, and S. K. Kulkarni, "Influence of biomass and gold salt concentration on nanoparticle synthesis by the tropical marine yeast Yarrowia lipolytica NCIM 3589," Colloids and Surfaces B: Biointerfaces, vol. 74, no. 1, pp. 309-316, 2009.

[28] S. S. Shankar, A. Rai, A. Ahmad, and M. Sastry, "Rapid synthesis of $\mathrm{Au}, \mathrm{Ag}$, and bimetallic Au core-Ag shell nanoparticles using Neem (Azadirachta indica) leaf broth," Journal of Colloid and Interface Science, vol. 275, no. 2, pp. 496-502, 2004.
[29] S. Li, Y. Shen, A. Xie et al., "Green synthesis of silver nanoparticles using Capsicum annuum L. extract," Green Chemistry, vol. 9, no. 8, pp. 852-858, 2007.

[30] A. Nabikhan, K. Kandasamy, A. Raj, and N. M. Alikunhi, "Synthesis of antimicrobial silver nanoparticles by callus and leaf extracts from saltmarsh plant, Sesuvium portulacastrum L.," Colloids and Surfaces B: Biointerfaces, vol. 79, no. 2, pp. 488-493, 2010.

[31] H. Xu and M. Käll, "Surface-plasmon-enhanced optical forces in silver nanoaggregates," Physical Review Letters, vol. 89, no. 24, Article ID 246802, 2002.

[32] J. L. Gardea-Torresdey, E. Gomez, J. R. Peralta-Videa, J. G. Parsons, H. Troiani, and M. Jose-Yacaman, "Alfalfa sprouts: a natural source for the synthesis of silver nanoparticles," Langmuir, vol. 19, no. 4, pp. 1357-1361, 2003.

[33] M. Vijayakumar, K. Priya, F. T. Nancy, A. Noorlidah, and A. B. A. Ahmed, "Biosynthesis, characterisation and anti-bacterial effect of plant-mediated silver nanoparticles using Artemisia nilagirica," Industrial Crops and Products, vol. 41, no. 1, pp. 235240, 2013.

[34] A. N. Mishra, S. Bhadauria, M. S. Gaur, R. Pasricha, and B. S. Kushwah, "Synthesis of gold nanoparticles by leaves of zero-calorie sweetener herb (Stevia rebaudiana) and their nanoscopic characterization by spectroscopy and microscopy," International Journal of Green Nanotechnology: Physics and Chemistry, vol. 1, no. 2, pp. 118-124, 2010.

[35] N. Akhtar, A. Mehmood, B. A. Khan, T. Mahmood, H. M. S. Khan, and T. Saeed, "Exploring cucumber extract for skin rejuvenation," African Journal of Biotechnology, vol. 10, no. 7, pp. 1206-1216, 2011.

[36] A. Mekonnen, T. Sidamo, K. Asres, and E. Engidawork, "In vivo wound healing activity and phytochemical screening of the crude extract and various fractions of Kalanchoe petitiana A. Rich (Crassulaceae) leaves in mice," Journal of Ethnopharmacology, vol. 145, no. 2, pp. 638-646, 2013.

[37] J. A. O. Olugbuyiro, K. A. Abo, and O. O. Leigh, "Wound healing effect of Flabellaria paniculata leaf extracts," Journal of Ethnopharmacology, vol. 127, no. 3, pp. 786-788, 2010. 

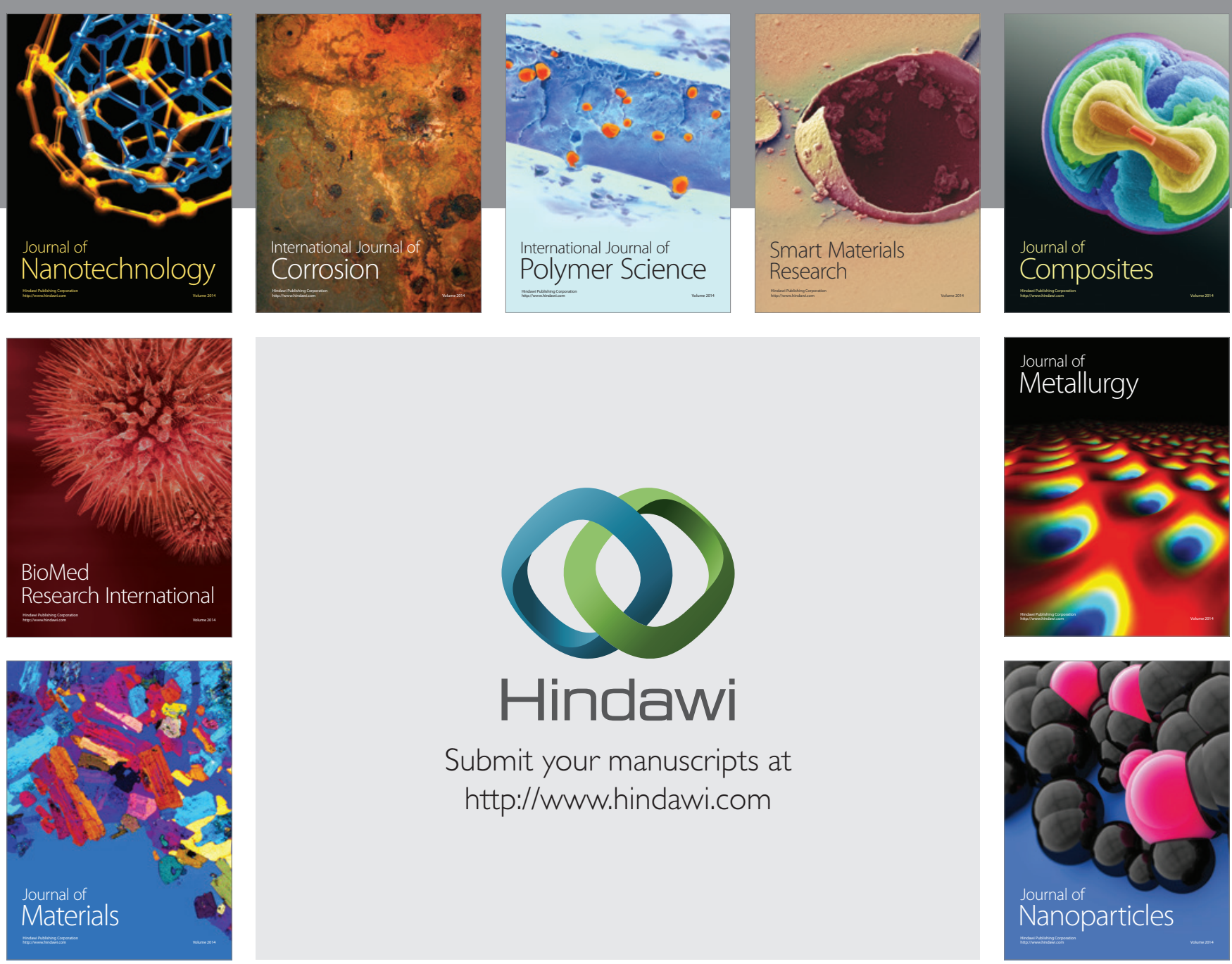

Submit your manuscripts at http://www.hindawi.com
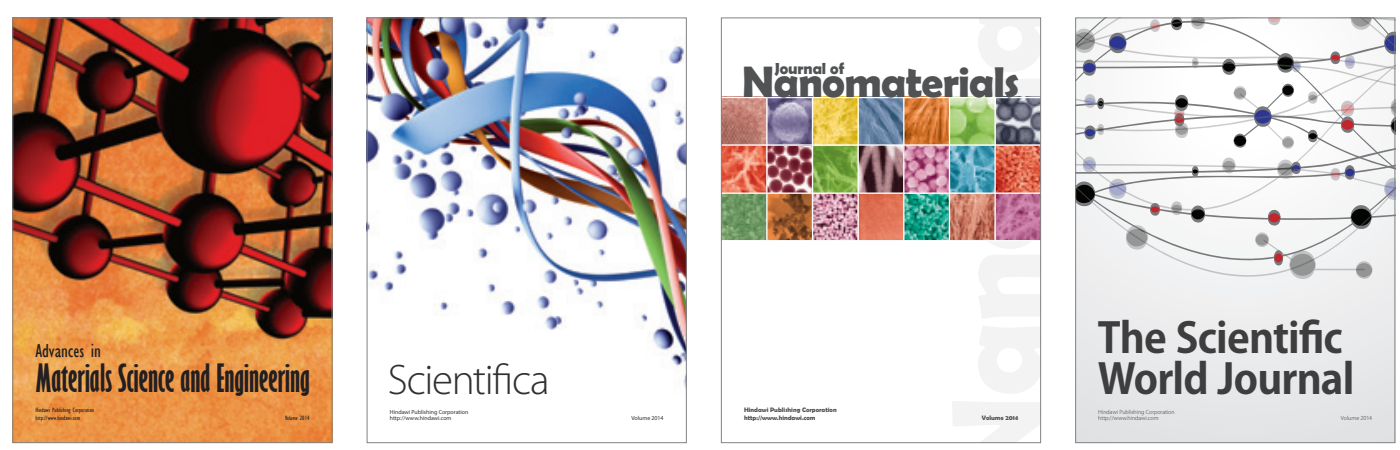

\section{The Scientific World Journal}
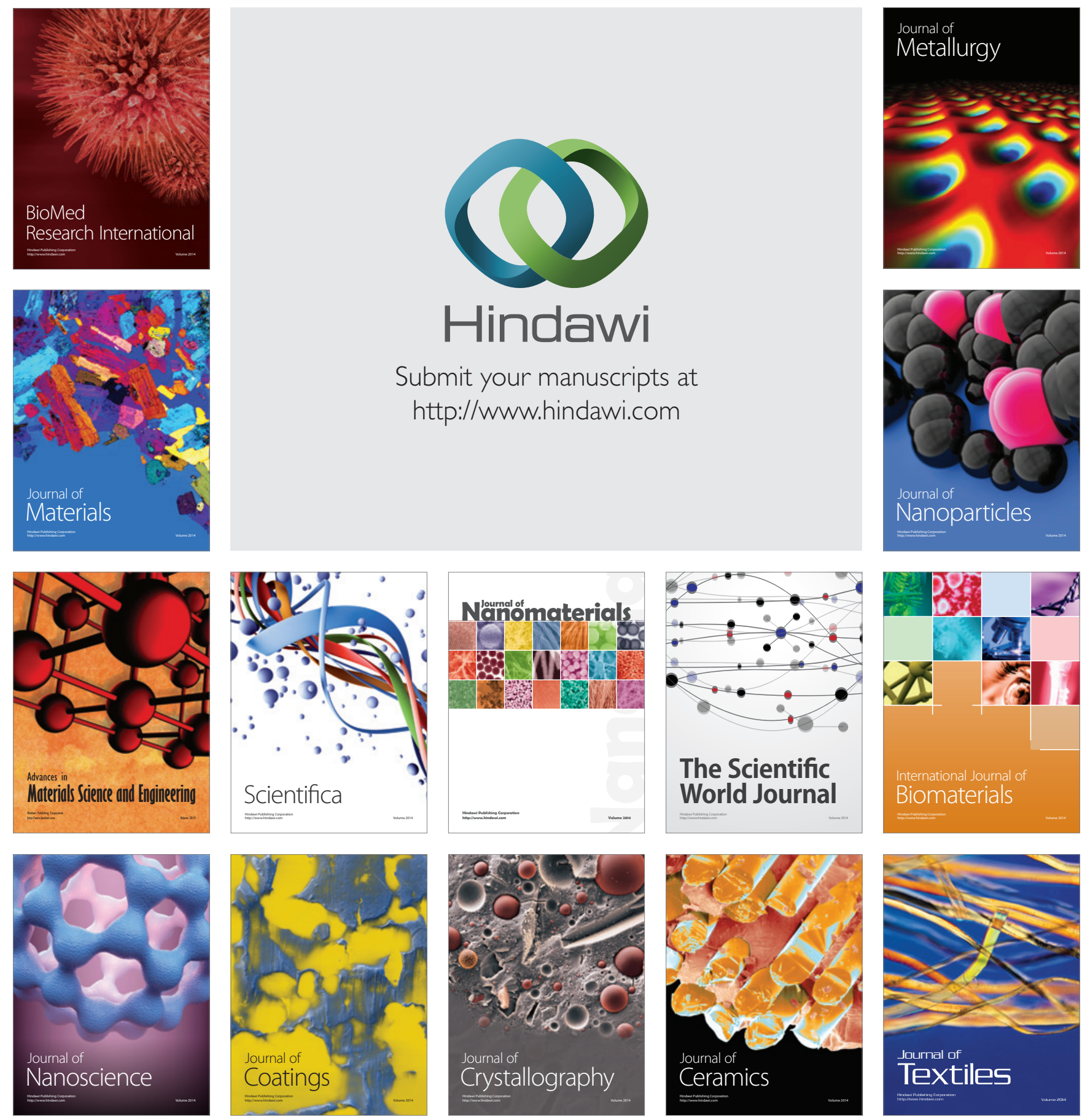